Commun. Math. Phys. 125, 71-79 (1989)

Communications in

Mathematical

Physics

(C) Springer-Verlag 1989

\title{
From PCA's to Equilibrium Systems and Back*
}

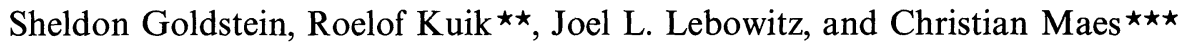 \\ Department of Mathematics and Physics, Rutgers University, New Brunswick, NJ 08903, USA
}

Dedicated to Roland Dobrushin

\begin{abstract}
Stationary measures for probabilistic cellular automata (PCA's) in $d$ dimensions give rise to space-time histories whose statistics may naturally be described by Gibbs states in $d+1$ dimensions for an interaction energy $\mathscr{H}$ obtained from the PCA. In this note we study the converse question: Do all Gibbs states for this $\mathscr{H}$ correspond to statistical space-time histories for the PCA? Our main result states that the answer is yes, at least for translation invariant or periodic Gibbs states. Thus ergodicity questions for PCA's can, at least partially, be formulated as questions of uniqueness of Gibbs states.
\end{abstract}

\section{Introduction}

Probabilistic Cellular Automata (PCA's) - which include the deterministic ones as special cases - are interesting subjects of study both mathematically and as models of various phenomena [1-6]. In this note we study some aspects of the connection between PCA's in $d$ dimensions and statistical mechanical Gibbs states in $(d+1)$ dimensions $[7,8]$, the extra dimension being the discrete time. Our objective is to facilitate the transfer of information, particularly rigorous results, between these subjects.

The state space of our PCA is the set of spin configurations $\sigma$ on a $d$-dimensional lattice, at each site of which there is a spin variable which takes on a discrete set of values. We shall consider for concreteness the lattice $\mathbb{Z}^{d}$ and Ising spin variables taking on the values of \pm 1 . The PCA describes a stochastic discrete time evolution of the spin configuration on $\mathbb{Z}^{d}$. We denote the value of the spin at site $i \in \mathbb{Z}^{d}$ at time $n \in \mathbb{Z}$ by $\sigma_{n, i}= \pm 1$, and write $\underline{\sigma}_{n}=\left\{\sigma_{n, i}\right\}$ for the spin configuration at time $n$.

\footnotetext{
* Research supported by NSF Grants DMR-86-12369 and DMS-85-12505

$\star \star$ Permanent address: Erasmus University, Faculteit Bedijfskunde Rotterdam

$\star \star \star$ Aspirant N.F.W.O., Instituut voor Theoretische Fysika, K.U. Leuven, Celestijnenlaan 200D, B-3030 Leuven, Belgium
} 
Given the spin configuration $\sigma_{n-1}$, the probability distribution of the configuration $\underline{\sigma}_{n}$ at time $n$ is given by a product of the probabilities of the spins $\sigma_{n, i}$, which we denote by $p_{i}\left(d \sigma_{n, i} \mid \sigma_{n-1}\right)$. That is, we have

$$
P\left(d \underline{\sigma}_{n} \mid \underline{\sigma}_{n-1}\right)=\prod_{i \in \mathbb{Z}^{d}} p_{i}\left(d \sigma_{n, i} \mid \underline{\sigma}_{n-1}\right)
$$

for the overall "transition probability" $P$, which may be regarded as defining the PCA. The $p_{i}$ 's satisfy the obvious normalization condition

$$
\sum_{\sigma_{n, i}= \pm 1} p_{i}\left(\sigma_{n, i} \mid \sigma_{n-1}\right)=1
$$

We assume that $p_{i}\left(\sigma_{n, i} \mid \cdot\right)$ is translation invariant and depends only on spins at time $n-1$ which are in some finite neighborhood $U(i)$ of the site $i$. Thus $U(i)=U+i$ for some fixed $U(=U(0))$ and

$$
p_{i}\left(\sigma_{n, i} \mid \underline{\sigma}\right)=p\left(\sigma_{n, i} \mid \underline{\sigma}_{U(i)}\right)
$$

where $\underline{\sigma}_{U(i)}$, the configuration of the spins $\underline{\sigma}$ in the set $U(i)$, should be regarded as identified with its translate to a configuration of spins on $U$. Our PCA thus defines a translation-invariant homogeneous discrete time (one-step) Markov process on $\{-1,1\}^{\mathbb{Z}^{d}}$ with spins simultaneously and independently updated.

It is now natural to consider $\sigma=\left\{\sigma_{n, i}\right\}$ as defining a spin configuration on the space-time lattice $\mathbb{Z}^{d+1}$; we write $x=(n, i)$ for a typical site in this lattice, we let $\mathbb{Z}_{N}^{d}$ denote the $d$-dimensional layer corresponding to $n=N$, and, with some abuse of notation, we let $U(x)=\{(n-1, j) \mid j \in U(i)\} \subset \mathbb{Z}_{n-1}^{d}$ be $U(i)$ at time $n-1$. For any set $\Gamma \subset \mathbb{Z}^{d+1}$ we write $\sigma_{\Gamma}$ for the restriction of the space-time spin configuration $\sigma$ to $\Gamma$.

If $v$ is a probability measure on the state space of the PCA and we "start" the time evolution with measure $v$ on the layer $\mathbb{Z}_{-N}$, the Markov transition rates (1.1) define a measure on the set of configurations on $\bigcup_{n \geqq-N} \mathbb{Z}_{n}^{d}$; if in addition $v$ is stationary $(v P=v)$ or periodic $\left(v P^{k}=v\right.$ for some $\left.k \geqq 1\right)$ for the time evolution, then the $N \rightarrow \infty$ limit, along the subsequence $N=k M$ if $v$ has period $k$, produces a measure $\mu_{v}$ on the set of space-time configurations $\{\sigma\}$. (By compactness, the PCA has at least one stationary probability measure $v$.)

The measure $\mu_{v}$ is an example of a Markov chain measure for the PCA, sometimes called simply a Markov chain, that is, a measure $\mu$ on space-time histories (here spin configurations $\sigma$ on $\mathbb{Z}^{d+1}$ ) satisfying

$$
\mu\left(d \underline{\sigma}_{n} \mid \underline{\sigma}_{<n}\right)=P\left(d \underline{\sigma}_{n} \mid \underline{\sigma}_{n-1}\right)
$$
for all $n \in \mathbb{Z}$, where $\underline{\sigma}_{<n}$ denotes the restriction of $\sigma$ to $\bigcup_{k<n} \mathbb{Z}_{k}^{d}$. Equation (1.4) is
equivalent to

$$
\mu\left(\sigma_{\Lambda} \mid \underline{\sigma}_{<n}\right)=\prod_{x \in \Lambda} p\left(\sigma_{x} \mid \sigma_{U(x)}\right)
$$

for all $n \in \mathbb{Z}$ and all finite $\Lambda \subset \mathbb{Z}_{n}^{d}$.

We assume that our PCA has no traps, i.e. that $p\left(\sigma_{x} \mid \sigma_{U(x)}\right)>0$. Then following Domany $[9,10]$, we associate with our PCA the Hamiltonian

$$
\mathscr{H}(\sigma)=\sum_{x} H\left(\sigma_{x}, \sigma_{U(x)}\right),
$$


where the single site energy $H\left(\sigma_{x}, \sigma_{U(x)}\right)$ is defined by

$$
\exp \left[-H\left(\sigma_{x}, \sigma_{U(x)}\right)\right]=p\left(\sigma_{x} \mid \sigma_{U(x)}\right) .
$$

Note that in terms of $H$ the Markov chain condition (1.5) becomes

$$
\mu\left(\sigma_{\Lambda} \mid \underline{\sigma}_{<n}\right)=\exp \left[-\sum_{x \in \Lambda} H\left(\sigma_{x}, \sigma_{U(x)}\right)\right]
$$

for all $n \in \mathbb{Z}$ and all finite $\Lambda \subset \mathbb{Z}_{n}^{d}$.

Now it is easy to see that any Markov chain measure $\mu$ for our PCA is a Gibbs measure for $\mathscr{H}$, i.e. for the translation invariant interaction $\Phi=\left\{\Phi_{A}\right\}$ with $A$ of the form $A=\{x\} \cup U(x), x \in \mathbb{Z}^{d+1}$, and $\Phi_{A}=H\left(\sigma_{x}, \sigma_{U(x)}\right)$. In fact, consider a finite region $V \subset \mathbb{Z}^{d+1}$ for which there exists a $T \in \mathbb{Z}$ such that whenever $x \in V$ either $U(x) \in V$ or $U(x) \in \mathbb{Z}_{T}^{d}$. The simplest such $V$ 's are the (space-time) translates of the negative cone $C_{N}=\mathbb{Z}^{d+1} \cap\{n \tilde{U}(o) \mid 0 \leqq n \leqq N\}$ of height $N$ generated by the convex hull $\tilde{U}(o)$ of $U(o)$, where $o$ is the origin of $\mathbb{Z}^{d+1}, C_{N}$ is the set of lattice sites in the convex hull of points influencing the origin up to $N$ steps back in time. We have by (1.8) that

$$
\mu\left(\sigma_{V}\right)=\int \mu\left(d \underline{\sigma}_{T}\right) \exp \left[-\sum_{x \in V} H\left(\sigma_{x}, \sigma_{U(x)}\right)\right]
$$

(where $\sigma_{U(x)}$ for $x \in \mathbb{Z}_{T+1}^{d}$ agrees with $\underline{\sigma}_{T}$ ) so that $\mu\left(\sigma_{V}\right)$ is just the Gibbs state in the volume $V$ with boundary condition given by $\mu\left(d \underline{\sigma}_{T}\right)$ on the base of $V$ and free boundary conditions on the remaining "sides" of $V$. Note that by virtue of the "normalization" (1.2), (1.3), and (1.7) the partition function $Z\left(V, \underline{\sigma}_{T}\right)$ in $V$ with fixed boundary condition $\underline{\sigma}_{T}$ on the base of $V$ satisfies

$$
Z\left(V \mid \underline{\sigma}_{T}\right)=\sum_{\sigma_{V}} \exp \left[-\sum_{x \in V} H\left(\sigma_{x}, \sigma_{U(x)}\right)\right]=1,
$$

so that the interaction arising from the PCA has free energy

$$
f=\lim _{V \nearrow \mathbb{Z}^{d+1}}|V|^{-1} \ln Z\left(V \mid \underline{\sigma}_{T}\right)=0 .
$$

(Recall that, in fact, the free energy does not depend upon the particular sequence whenever $V \nearrow \mathbb{Z}^{d+1}$ in the sense of van Hove, nor upon boundary conditions $[7,8]$.)

Notice that the reciprocal temperature $\beta$ which usually multiplies the energy in the exponent of (1.9) has been absorbed in $H$, which, defined through (1.7), will in specific examples contain various "interaction parameters" $\underline{J}$ which can be identified with magnetic field $h$, pair interactions, etc. .... It follows from (1.10) that the free energy is trivially analytic in the $J$ 's even when the stationary state $v$ for the PCA, and hence $\mu_{v}$, undergoes a "phase transition" as the parameters in the PCA are varied (cf. Domany et al. $[9,10])$. Since the coexistence of more than one phase for the Hamiltonian $\mathscr{H}$ implies that there are directions, in the space of interactions, along which the free energy has a cusp $[7,8]$, varying the PCA parameters must sweep out a rather special surface in this space. In fact there are simple examples [6] in which it can be shown that the entropy and energy are separately analytic in the PCA parameters when there is a phase transition.

We shall investigate here the converse problem of whether every Gibbs state $\mu$ for $\mathscr{H}(1.6)$ is a Markov chain measure for our PCA. If this were true then we could 
conclude, in particular, that i) if there is more than one Gibbs state invariant under "time" translations then the PCA has more than one stationary $v$, and ii) if there is more than one Gibbs state then it is not the case that the PCA is ergodic in the sense that there exists a stable stationary $v$ such that for every initial state $\tilde{v}, \tilde{v} P^{n} \rightarrow v$ as $n \rightarrow \infty$.

This question arises because, as is well known [5], the Gibbs states are precisely the solutions to the DLR equations

$$
\mu\left(\sigma_{V} \mid \sigma_{V c}\right)=\exp \left[-\sum_{x} H\left(\sigma_{x}, \sigma_{U(x)}\right)\right] / Z\left(V \mid \sigma_{V^{c}}\right)
$$

for all finite $V \subset \mathbb{Z}^{d+1}$, where the summation $\sum_{x}$ is over all $x$ for which $(\{x\} \cup U(x))$ $\cap V \neq \phi$. In other words all (extremal) Gibbs states may be obtained as infinite volume limits $V \nearrow \mathbb{Z}^{d+1}$ of the finite volume Gibbs states defined by (1.11); here the choice of $V$ is immaterial, and we may, if we wish, use only the conical $V$ 's involved in the demonstration that Markov chains are Gibbs states, but we must allow for general boundary conditions - containing all interactions of the spins in $V$ with those in the exterior of $V$-instead of using just free boundary conditions on the "sides" of $V$. Note that even if $\sigma_{T}$ is compatible with $\sigma_{V c}$, the measure describing the evolution of our PCA starting with the fixed configuration $\sigma_{T}$ at time $T$ (the base of $V$ lying on $\mathbb{Z}_{T}^{d}$ ) need not agree with (1.11) on $V$, which describes the conditional distribution in $V$ for the PCA given the spins in the past and the future (i.e. the "sides") of $V$. Thus the problem is whether in the thermodynamic limit these Gibbs measures have transition probabilities given by the PCA (1.8). Now there seems to be no a priori reason why this should be so; indeed, in a more general setting there are counterexamples - see Remark 4 in Sect. 3. It is in fact generally very difficult to prove any results about the number of Gibbs states for a given Hamiltonian except at high temperatures or in one dimension. What we require here seems in a way even more difficult since by their definition the PCA states have the global markov property in the time direction - a property which is also very difficult to prove for any Gibbs state in the region of non-uniqueness, see Remark 5 in Sect. 3. It is therefore surprising that the answer for Hamiltonians of the form (1.6) is positive for all those Gibbs states which are translation invariant or periodic in $\mathbb{Z}^{d+1}$.

\section{Results and Proofs}

We now give our results. Define the lexicographic order of $\mathbb{Z}^{d+1}$ by putting $x<y$ for $x=\left(x_{1}, x_{2}, \ldots, x_{d+1}\right), y=\left(y_{1}, y_{2}, \ldots, y_{d+1}\right) \in \mathbb{Z}^{d+1}$ if $x_{1}<y_{1}$, or $x_{1}=y_{1}$ and $x_{2}<y_{2}$, or ....

Let

$$
P \equiv\left\{x \in \mathbb{Z}^{d+1}: x<o\right\}
$$

consist of the sites in $\mathbb{Z}^{d+1}$ which precede the origin in this order. Note that the "temporal past" $\bigcup_{n<0} \mathbb{Z}_{n}^{d} \subset P$. 
Theorem. Let $\mu$ be a translation invariant Gibbs state for the interaction $\mathscr{H}$ defined in (1.6) and (1.7). Then

$$
\mu\left(\sigma_{o} \mid \sigma_{P}\right)=\exp \left[-H\left(\sigma_{o}, \sigma_{U(o)}\right)\right]
$$

is the distribution on $\sigma_{o}$ conditioned on the configuration $\sigma_{P}$ in $P$.

Note that the Markov chain condition (1.8) implies (2.2). Conversely, since according to (2.2) $\mu\left(\sigma_{o} \mid \sigma_{P}\right)$ does not depend upon the spins in $\mathbb{Z}_{o}^{d} \backslash\{o\}$, it follows from (2.2) that $\mu$ satisfies the Markov chain condition (1.8).

Corollary. The (space-time) translation-invariant Gibbs states for $\mathscr{H}$ are the translation invariant stationary Markov chains for $P$.

Remark. It follows immediately from the corollary that non-uniqueness of the translation-invariant Gibbs state is equivalent to non-uniqueness of the stationary translation-invariant measure for the PCA. (Of course non-uniqueness of the stationary measure or, more generally, of the Markov chain measure for the PCA implies non-uniqueness of the Gibbs measure.)

In Sect. 3 we will show how to extend this result to periodic states and briefly discuss the global Markov property which is related to our results.

The proof of the theorem makes use of entropy and conditional entropy. For any probability measure $\mu$ on $\Omega=\{-1,1\}^{\mathbb{Z}^{d+1}}$, the entropy $S_{V}(\mu)$ associated with a finite volume $V \subset \mathbb{Z}^{d+1}$ is defined by

$$
S_{V}(\mu)=-\sum_{\sigma_{V}} \mu\left(\sigma_{V}\right) \ln \mu\left(\sigma_{V}\right) .
$$

If $\mu$ is translation invariant its entropy density $s(\mu)$ is then given by the limit (via a proper sequence of volumes $V$, say hypercubes)

$$
s(\mu)=\lim _{V \nearrow \mathbb{Z}^{d+1}} S_{V}(\mu) /|V| .
$$

When $V_{1}$ and $V_{2}$ are finite volumes then

$$
S_{V_{1} \cup V_{2}}(\mu)=S_{V_{1}}(\mu)+S_{V_{2} \mid V_{1}}(\mu),
$$

where

$$
S_{V_{2} \mid V_{1}}(\mu)=\int \mu\left(d \sigma_{V_{1}}\right) S_{V_{2}}\left(\mu\left(\cdot \mid \sigma_{V_{1}}\right)\right) .
$$

Furthermore the conditional entropy $S_{V_{2} \mid V_{1}}(\mu)$ is non-increasing in the volume $V_{1}$ ("monotonicity of conditional entropy"), and is well defined by (2.6) for $V_{1}$ infinite.

As the next lemma tells us, the entropy density can be related to conditional entropy.

Lemma. Let $\mu$ be a translation invariant probability measure on $\Omega$. Then

$$
s(\mu)=S_{\{o\} \mid P}(\mu) .
$$

This lemma, which is standard, and the variational principle are the main ingredients in the proof of the theorem.

Proof of the Theorem. We start by considering a local version of the variational principle. Consider a fixed configuration $\sigma_{U(o)}$ on $U(o)$, and let $W\left(\sigma_{o}\right)$ be the unique 
equilibrium distribution on $\sigma_{o}$ for the interaction $H\left(\sigma_{o}, \sigma_{U(o)}\right)$; that is,

$$
W\left(\sigma_{o}\right)=\exp \left[-H\left(\sigma_{o}, \sigma_{U(o)}\right)\right] .
$$

Then for any probability distribution $\varrho\left(\sigma_{o}\right)$ on $\sigma_{o}$, the corresponding free energy

$$
\sum_{\sigma_{o}} \varrho\left(\sigma_{o}\right) H\left(\sigma_{o}, \sigma_{U(o)}\right)+\sum_{\sigma_{o}} \varrho\left(\sigma_{o}\right) \ln \varrho\left(\sigma_{o}\right)
$$

attains its minimum value precisely when $\varrho\left(\sigma_{o}\right)=W\left(\sigma_{o}\right)$. Moreover, because of the "normalization" condition (1.2), this value is 0 .

Next we turn to the full variational principle. Let $\mu$ be a translation invariant probability measure on $\Omega$. The energy density $e(\mu)$ of $\mu$ is given by

$$
e(\mu)=\int \mu(d \sigma) H\left(\sigma_{o}, \sigma_{U(o)}\right)=\int \mu\left(d \sigma_{P}\right) \sum_{\sigma_{o}} \mu\left(\sigma_{o} \mid \sigma_{P}\right) H\left(\sigma_{o}, \sigma_{U(o)}\right) .
$$

Therefore, using the lemma, the free energy may be written as

$$
e(\mu)-s(\mu)=\int \mu\left(d \sigma_{P}\right)\left[\sum_{\sigma_{o}} \mu\left(\sigma_{o} \mid \sigma_{P}\right) H\left(\sigma_{o}, \sigma_{U(o)}\right)-S_{\{o\}}\left(\mu\left(\cdot \mid \sigma_{P}\right)\right)\right] .
$$

It follows from the local variational principle that the quantity in the bracket [ ] has its minimum value, which is zero for all $\sigma_{P}$, when (2.2) is satisfied. Thus a translation invariant probability measure $\mu$ minimizes the free energy, i.e. is an equilibrium (Gibbs) state, precisely when (2.2) is satisfied. [Note that it also follows directly from (2.11) that the interaction arising from the PCA has free energy zero.]

Though it is fairly well known, see for instance [11], for the sake of completeness we give a

Proof of the Lemma. Put $V_{n}=\left\{x \in \mathbb{Z}^{d+1}|| x_{i} \mid \leqq n, i=1, \ldots, d+1\right\}, \quad V_{n}(y)$ $=V_{n} \cap\left\{x \in \mathbb{Z}^{d+1} \mid x<y\right\}$ and let $\bar{s}=S_{\{0\} \mid P}(\mu)$. Since $\mu_{\{0\}}\left(\cdot \mid \sigma_{V_{n}(o)}\right) \rightarrow \mu_{\{0\}}\left(\cdot \mid \sigma_{P}\right)$ as $n \rightarrow \infty$, it follows from the monotonicity of the conditional entropy that

$$
\bar{s}=\inf _{n} S_{\{0\} \mid V_{n}(o)}(\mu) .
$$

Moreover, since $\mu$ is translation invariant,

$$
\bar{s} \leqq S_{\{y\} \mid V_{n}(y)}(\mu) .
$$

Now using (2.5) iteratively,

$$
S_{V_{n}}(\mu)=\sum_{y \in V_{n}} S_{\{y\} \mid V_{n}(y)}(\mu),
$$

so that

$$
s(\mu)=\lim _{n \rightarrow \infty} S_{V_{n}}(\mu) /\left|V_{n}\right| \geqq \bar{s} .
$$

On the other hand take $N \in \mathbb{N}$ and consider

$$
W_{n}=\left\{y \in V_{n} \mid \exists \text { a translation } \tau \text { such that } \tau V_{n}(y) \supset V_{N}(o)\right\} .
$$

Then, for $y \in W_{n}$,

$$
S_{\{y\} \mid V_{n}(y)}(\mu) \leqq S_{\{o\} \mid V_{N}(o)}(\mu),
$$


and

$$
\lim _{n}\left|W_{n}\right| /\left|V_{n}\right|=1
$$

Thus, combining (2.14), (2.16), and (2.17), we find that

$$
s(\mu)=\lim _{n} S_{V_{n}}(\mu) /\left|V_{n}\right| \leqq S_{\{o\} \mid V_{N}(o)}(\mu)
$$

for any $N \in \mathbb{N}$, so that by (2.12)

$$
s(\mu) \leqq \bar{s} .
$$

Thus, in view of $(2.15), s(\mu)=\bar{s}$, and the proof is complete.

\section{Concluding Remarks}

1. Our analysis applies essentially verbatium to the case of spin variables taking on any finite number of values and presumably applies as well to the case of compact spins. We can also, without difficulty, make other generalizations of our PCA's. We list two: a) Not all spins are updated simultaneously; e.g. first update even sites then odd ones (as done by Domany $[6,7]$ ). b) Divide the lattice $\mathbb{Z}^{d}$ into disjoint sets $A_{k}, \cup A_{k}=\mathbb{Z}^{d}$, connected by some transitive symmetry group, and let the spin configurations on different $A_{k}$ 's be simultaneously and independently updated according to $p\left(\sigma_{A_{k}} \mid \sigma_{U_{A_{k}}}\right)$. E.g. for $d=1$, let $A_{k}=(2 k, 2 k+1)$, a pair of adjacent sites, and let $U_{A_{k}}$ be a set of (nearby) sites at the preceding time. The extension to case a) is trivial while case b) is simply handled by considering the $\sigma_{A_{k}}$ as spin variables taking on $2^{\left|A_{k}\right|}$ values.

Another generalization is the following: Let $U(x)=U(o)+x$, where $U(o)$ is a finite set of points all of which precede $o$ in the lexicographic order of $\mathbb{Z}^{d+1}$, and let $H\left(\sigma_{x}, \sigma_{U(x)}\right)$ be a translation invariant interaction satisfying the "normalization" condition $\sum_{\sigma_{x}} \exp \left[-H\left(\sigma_{x}, \sigma_{U(x)}\right)\right]=1$. By the same proof the theorem still holds. However, the translation-invariant Gibbs states, though satisfying (2.2), will not in general correspond to PCA's with synchronous independent updating, nor, for that matter, need they define Markov chains, even for nearest neighbor interactions. However, for suitably chosen $U(o)$ the "dynamics" defined by (2.2) can be thought of as corresponding to the infinite volume limit of a discrete time PCA with asynchronous lexicographical updating.

2. The theorem holds for periodic Gibbs states as well. By a periodic Gibbs state we mean a Gibbs state which is invariant under a subgroup $G$ of $\mathbb{Z}^{d+1}$ that has finite index in $\mathbb{Z}^{d+1}$; that is, $\left|\mathbb{Z}^{d+1} / G\right|<\infty$. Let $\mu^{\prime}$ be a periodic Gibbs state. The argument that (2.2) holds for $\mu^{\prime}$ is roughly as follows. There exists a translation invariant Gibbs state $\mu$ such that $\mu^{\prime}$ is absolutely continuous with respect to $\mu$. The Radon-Nikodym derivative $\frac{d \mu^{\prime}}{d \mu}$ is a $G$-invariant function [in $\left.L^{1}(d \mu)\right]$ and $G$-invariant functions depend only on $\sigma_{P}(\mu \bmod 0)$. From this and the fact that (2.2) holds for $\mu$ it readily follows that (2.2) holds also for $\mu^{\prime}$ (see [12] for more details). 
3. Consider the case $d=1$. In view of the corollary one is led to consider proving (or disproving) the longstanding open question of the ergodicity of 1-dimensional PCA's (with positive rates), at least for translation invariant ones, by considering the associated two-dimensional Gibbs states. However the analysis of the two-dimensional Gibbs states seems as hard as a direct PCA analysis.

4. We have shown that within the class of space-time translation-invariant (or periodic) measures, Markov chains for a PCA and Gibbs states for the corresponding interaction are precisely the same objects. The question of whether symmetry properties, which allow us to use the variational principle, are required for this identification remains open, though it appears unlikely that the identification is of universal validity. (For a counterexample in 0 space dimensions but with $\mathbb{N}$-valued spin variables, see Chap. 10 of [17].)

5. By definition, Gibbs states satisfy the local Markov property: for every finite volume $V, \mu\left(\sigma_{V} \mid \sigma_{V c}\right)=\mu\left(\sigma_{V} \mid \sigma_{\partial V}\right)$, where $\partial V$ is the boundary of $V$, with thickness equal to the range of the interaction. An interesting question in equilibrium statistical mechanics is whether a similar Markov property holds for half-spaces where the boundary is $\mathbb{Z}_{n}^{d}$, an example of a global Markov property [12-16]. Even for nearest neighbor interactions, examples are known for which this property does not hold $[14,15]$. However, in view of Eq. (1.8) the Gibbs states we consider in this paper satisfy even more than a global Markov property: the relevant conditional probabilities are given by a specific local function (independent of the particular Gibbs state).

\section{References}

1. Dobrushin, R.L.: The description of a random field by means of conditional probabilities and its regularity. Theor. Prob. Appl. 13, 201 (1968)

Dobrushin, R.L.: Markov processes with a large number of locally interacting components: existence of a limit process and its ergodicity. Prob. Inform. Trans. 7, 149-164 (1971)

Dobrushin, R.L., Pjatetski-Shapiro, I.I., Vasilyev, N.B.: Markov processes in an infinite product of discrete spaces. Proceedings of the Soviet-Japanese Symposium in Probability Theory, Khavarovsk, U.S.S.R., (1969)

2. Toom, A.L.: Stable and attractive trajectories in random systems. In: Multicomponent random systems. Dobrushin, R.L., Sinai, Ya.G. (eds.). New York: Dekker 1980

3. Spitzer, F.: Infinite systems with locally interacting components. Ann. Probab. 9, 349-364 (1981)

4. Liggett, T.M.: Interacting particle systems. Berlin, Heidelberg, New York: Springer 1985

5. Bennet, C.H., Grinstein, G.: Role of irreversibility in stabilizing complex and nonergodic behavior in locally interacting discrete systems. Phys. Rev. Lett. 55, 657-660 (1985)

6. For some recent reviews see: Rujan, P.: Cellular automata and statistical mechanical models. J. Stat. Phys. 49, 139-222 (1987)

Georges, A., LeDoussal, P.: From equilibrium spin models to probabilistic cellular automata. Preprint, Lab. de Physique Theorique de l'Ecole Normal Superieure (1987); to appear in J. Stat. Phys. (1989)

Lebowitz, J.L., Maes, C., Speer, E.R.: Statistical mechanics of probabilistic cellular automata. Preprint, Rutgers University (1989); J. Stat. Phys. (to appear)

7. Israel, R.B.: Convexity in theory of lattice gas. Princeton, NJ: Princeton University Press 1979 Sinai, Y.G.: Theory of phase transitions: Rigorous results. Oxford: Pergamon Press 
8. Ruelle, D.: Statistical mechanics: Rigorous results. Reading, MA: Addison-Wesley Ruelle, D.: Thermodynamic formalism. Reading, MA: Addison-Wesley 1978

9. Domany, E.: Exact results for two- and three-dimensional Ising and Potts models. Phys. Rev. Lett. 52, 871-874 (1984)

10. Domany, E., Kinzel, W.: Equivalence of cellular automata to Ising models and directed percolation. Phys. Rev. Lett. 53, 311-314 (1984)

11. Katznelson, Y., Weiss, B.: Commuting measure-preserving transformations. Israel J. Math. 12, 161-173 (1972)

12. Goldstein, S., Kuik, R., Schlÿper, A.G.: Entropy and global Markov properties. Commun. Math. Phys. (in press)

13. Kuik, R.: Markov and stability properties of equilibrium states for nearest neighbor interactions. Commun. Math. Phys. 115, 529 (1988)

14. Goldstein, S.: Remarks on the global Markov property. Commun. Math. Phys. 74, 223-234 (1980)

15. Israel, R.B.: Some examples concerning the global Markov property. Commun. Math. Phys. 105, 669-673 (1986)

16. Föllmer, H.: On the global Markov property. In: Quantum fields, algebras, processes. Streit, L. (ed.), pp. 293-302. Wien, New York: Springer 1980

17. Georgii, H.: Gibbs measures and phase transitions. Berlin, New York: W. de Gruyter 1988

Communicated by A. Jaffe

Received January 9, 1989; in revised form May 2, 1989 
